\title{
Some numerical integration methods based on interpolation polynomials
}

\section{ANA MARIA ACU and DANIEL FLORIN SOFOnEA}

\begin{abstract}
.
In this paper we have considered the asymptotic expressions for remainder term of quadrature formulas of the interpolator type. We derive some corrected versions of the quadrature formulas of interpolatory type, which provide a better approximation accuracy than the original rules. A method to improve the degree of exactness of the quadrature formulas is also considered. A numerical example of the proposed method is given.
\end{abstract}

DEPARTMENT OF MATHEMATICS AND INFORMATICS

UNIVERSITY "LUCIAN BLAGA" OF SIBIU

DR. I. RAṬIU 5-7, 550012 SIBIU, ROMANIA

E-mail address: acuana77@yahoo.com

E-mail address: sofoneaflorin@yahoo.com

Received: 30.10.2011; In revised form: 02.09.2012; Accepted: 15.10.2012

2010 Mathematics Subject Classification. 26D15, 65D30.

Key words and phrases. Quadrature formulas of Gauss type, numerical integration, orthogonal polynomials.

Corresponding author: Ana Maria Acu; acuana77@yahoo.com 